\title{
On-treatment serum albumin level can guide long-term treatment in patients with cirrhosis and uncomplicated ascites
}

\section{Graphical abstract}

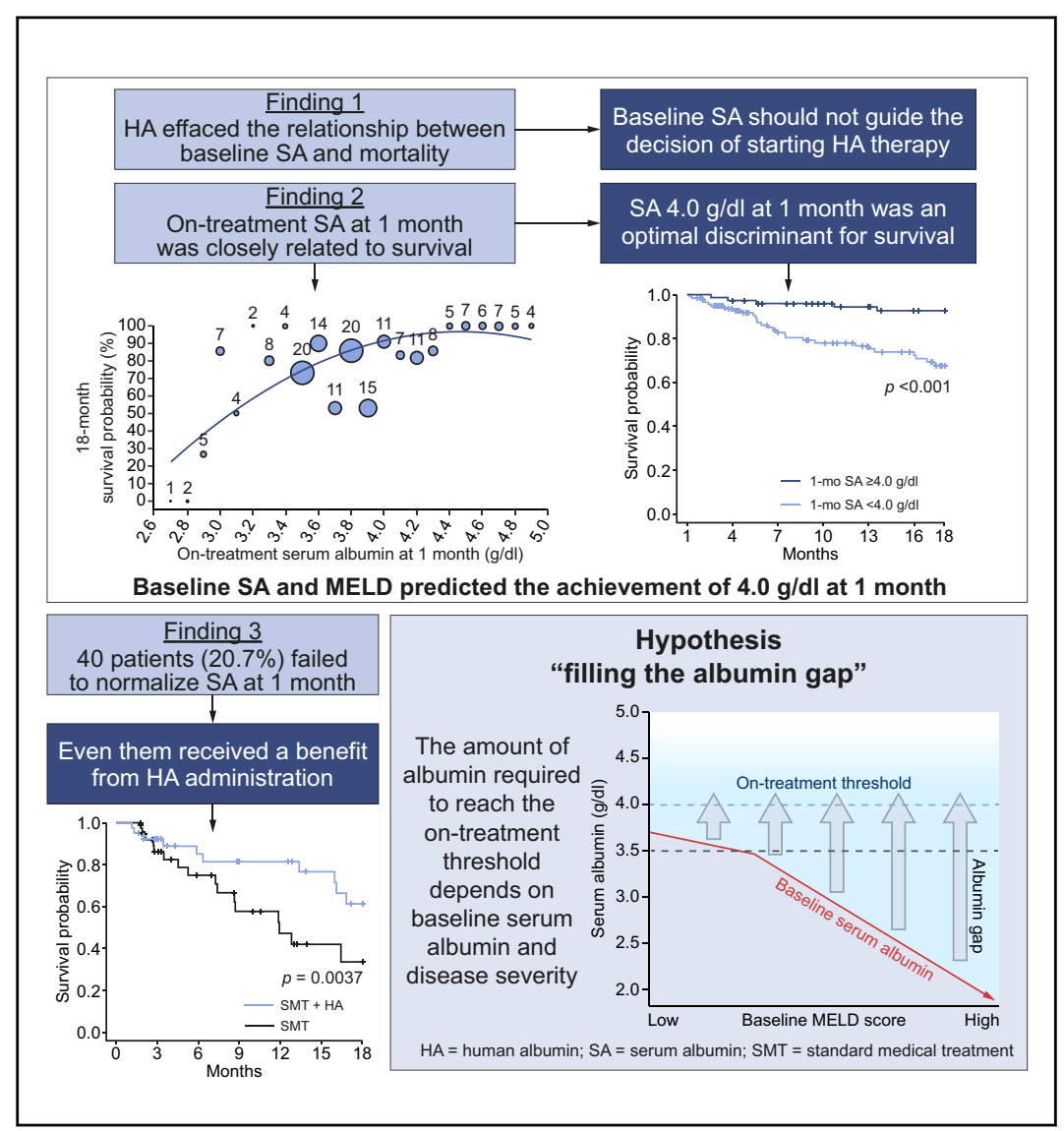

\section{Authors}

Paolo Caraceni, Manuel Tufoni, Giacomo Zaccherini, ...., Andrea Roncadori, Maurizio Baldassarre, Mauro Bernardi

\section{Correspondence}

mauro.bernardi@unibo.it (M. Bernardi).

\section{Lay summary}

The ANSWER study has shown that long-term albumin administration improves survival and prevents the occurrence of major complications in patients with cirrhosis and ascites. This study shows that the achievement of these beneficial effects is related to a significant increase in serum albumin concentration. Even though the best results follow the achievement of a serum albumin concentration of $4 \mathrm{~g} / \mathrm{dl}$, a survival benefit is also achieved in patients who fail to normalise serum albumin.

\section{Highlights}

- Baseline serum albumin per se should not guide the decision to start albumin therapy.

- 1-month on-treatment serum albumin levels predict survival and can be used to guide therapy.

- The serum albumin target threshold to be pursued is $4.0 \mathrm{~g} / \mathrm{dl}$.

- Baseline serum albumin and MELD score predict the achievement of this target.

- A survival benefit is seen even when on-treatment serum albumin does not normalize. 


\title{
On-treatment serum albumin level can guide long-term treatment in patients with cirrhosis and uncomplicated ascites
}

\author{
Paolo Caraceni ${ }^{1,2, \dagger}$, Manuel Tufoni ${ }^{1,2, \dagger}$, Giacomo Zaccherini ${ }^{1,2}$, Oliviero Riggio ${ }^{3}$, Paolo Angeli ${ }^{4}$, \\ Carlo Alessandria ${ }^{5}$, Sergio Neri ${ }^{6}$, Francesco G. Foschi ${ }^{7}$, Fabio Levantesi ${ }^{8}$, Aldo Airoldi ${ }^{9}$, \\ Loredana Simone $^{10}$, Gianluca Svegliati-Baroni ${ }^{11}$, Stefano Fagiuoli ${ }^{12}$, Giacomo Laffi ${ }^{13}$, \\ Raffaele Cozzolongo ${ }^{14}$, Vito Di Marco ${ }^{15}$, Vincenzo Sangiovanni ${ }^{16}$, Filomena Morisco ${ }^{17}$, \\ Pierluigi Toniutto ${ }^{18}$, Antonio Gasbarrini ${ }^{19}$, Rosanna De Marco $^{20}$, Salvatore Piano \\ Silvia Nardelli ${ }^{3}$, Chiara Elia $^{5}$, Andrea Roncadori ${ }^{21}$, Maurizio Baldassarre ${ }^{1,22}$, Mauro Bernardi ${ }^{1, *}$, \\ for the ANSWER Study Investigators
}

\begin{abstract}
${ }^{1}$ Department of Medical and Surgical Sciences, University of Bologna, Italy; ${ }^{2}$ Bologna University Hospital Authority St. Orsola-Malpighi Polyclinic; ${ }^{3}$ Department of Clinical Medicine, "Sapienza" University of Rome, Italy; ${ }^{4}$ Unit of Internal Medicine and Hepatology, Department of Medicine, University of Padua, Italy; ${ }^{5}$ Division of Gastroenterology and Hepatology, "Città della Salute e della Scienza" Hospital, University of Turin, Italy; ${ }^{6}$ Department of Clinical and Experimental Medicine, University of Catania, Italy; ${ }^{7}$ Internal Medicine, Hospital of Faenza, A.U.S.L. of Romagna, Italy; ${ }^{8}$ Internal Medicine, Hospital of Bentivoglio, A.U.S.L. of Bologna, Italy; ${ }^{9}$ Liver Unit, Department of Hepatology and Gastroenterology, Niguarda Hospital, Milan, Italy; ${ }^{10}$ Gastroenterology Unit, University Hospital, Ferrara, Italy; ${ }^{11}$ Department of Gastroenterology, Polytechnic University of Marche, Ancona, Italy; ${ }^{12}$ Gastroenterology and Transplant Hepatology, Papa Giovanni XXIII Hospital, Bergamo, Italy; ${ }^{13}$ Careggi University Hospital, University of Florence, Italy; ${ }^{14}$ Division of Gastroenterology, National Institute of Gastroenterology “S. De Bellis", Castellana Grotte (Bari), Italy; ${ }^{15}$ Unit of Gastroenterology and Hepatology, Biomedical Department of Internal and Specialistic Medicine, University of Palermo, Italy; ${ }^{16}$ A.O.R.N. dei Colli - "Cotugno" Hospital of Naples, Italy; ${ }^{17}$ Gastroenterology Unit,

Department of Clinical Medicine and Surgery, "Federico II" University of Naples, Italy; ${ }^{18}$ Hepatology and Liver Transplantation Unit,

Department of Medical Area, University of Udine, Italy; ${ }^{19}$ Gastroenterology, Gemelli Foundation, Catholic University, Rome, Italy;

${ }^{20}$ Gastroenterology Unit, Hospital of Cosenza, Italy; ${ }^{21}$ Cineca Interuniversity Consortium, Bologna, Italy; ${ }^{22}$ Center for Applied Biomedical Research (CRBA), University of Bologna, Italy
\end{abstract}

Background \& Aims: The ANSWER study reported that longterm albumin administration in patients with cirrhosis and uncomplicated ascites improves survival. During treatment, serum albumin increased within a month and remained stable thereafter. In this post hoc analysis, we aimed to determine whether on-treatment serum albumin levels could guide therapy.

Methods: Logistic regression was used to assess the association between baseline serum albumin and mortality, as well as to determine on-treatment factors associated with mortality and to predict the achievement of a given on-treatment serum albumin level. Survival was assessed by Kaplan-Meier estimates and second-order polynomial regression. Patients whose ontreatment serum albumin remained below normal were compared with a subset of patients from the control arm matched by principal score.

Results: Baseline serum albumin was closely associated with 18-month mortality in untreated patients; albumin treatment

\footnotetext{
Keywords: Cirrhosis; Ascites; Serum albumin; Survival; Complications. Received 28 January 2020; received in revised form 10 July 2020; accepted 17 August 2020; available online $x x x$

* Corresponding author. Address: Department of Medical and Surgical Sciences University of Bologna, S. Orsola-Malpighi University Hospital, Via Albertoni 15 40138 - Bologna, Italy. Tel.: +39 051308943, fax: +39 0512142930.

E-mail address: mauro.bernardi@unibo.it (M. Bernardi).

Guest Editor: Dominique Valla.

$\dagger$ Authors share co-first authorship.

\# Investigators of the Human Albumin for the Treatment of Ascites in Patients with Hepatic Cirrhosis (ANSWER) study are listed at the end of the manuscript. https://doi.org/10.1016/j.jhep.2020.08.021
}

almost effaced this relationship. On-treatment serum albumin and MELD-Na at month 1 were the sole independent variables associated with mortality. Second-order polynomial regression revealed that survival improved in parallel with increased 1-month on-treatment serum albumin. KaplanMeier estimations showed that any value of 1-month ontreatment serum albumin $(0.1 \mathrm{~g} / \mathrm{dl}$ intervals $)$ in the range $2.5-4.5 \mathrm{~g} / \mathrm{dl}$ discriminated patient survival. In the normal range of serum albumin, the best discriminant value was 4.0 $\mathrm{g} / \mathrm{dl}$. Compared to untreated patients, survival even improved in patients whose on-treatment serum albumin remained below normal.

Conclusion: Baseline serum albumin per se should not guide the decision to start albumin therapy. Conversely, 1-month ontreatment serum albumin levels are strongly associated with outcomes and could guide the use of albumin $-4.0 \mathrm{~g} / \mathrm{dl}$ being the target threshold. However, even patients whose serum albumin remains below normal benefit from long-term albumin administration.

Lay summary: The ANSWER study has shown that long-term albumin administration improves survival and prevents the occurrence of major complications in patients with cirrhosis and ascites. This study shows that the achievement of these beneficial effects is related to a significant increase in serum albumin concentration. Even though the best results follow the achievement of a serum albumin concentration of $4 \mathrm{~g} / \mathrm{dl}$, a survival benefit is also achieved in patients who fail to normalise serum albumin. 
(c) 2020 European Association for the Study of the Liver. Published by Elsevier B.V. All rights reserved.

\section{Introduction}

Whether long-term human albumin (HA) administration to patients with cirrhosis and ascites exerts beneficial effects is a longdebated issue. Two recent prospective randomised controlled trials addressed this matter. ${ }^{1,2}$ In the open-label ANSWER study, which included patients with cirrhosis and uncomplicated persistent ascites, the addition of HA to standard medical treatment (SMT) improved patient survival, eased the management of ascites, and lowered the incidence of major complications of cirrhosis compared to SMT alone. ${ }^{1}$ Conflicting findings emerged from the MACHT study, a placebo-controlled trial comparing the effects of SMT plus HA combined with the $\alpha_{1}$-adrenergic agonist midodrine with SMT alone in patients with cirrhosis and ascites on a waiting list for liver transplantation. In fact, no differences in the incidence of complications and mortality were seen between the 2 arms of the study. ${ }^{2}$

There are several possible reasons for the variation in results, including study design, severity of cirrhosis at enrolment and duration of HA treatment. However, a major difference between the 2 studies is related to the amount of HA administered. Indeed, after a loading dose of $40 \mathrm{~g}$ twice weekly for 2 weeks, 40 $\mathrm{g}$ of HA once a week were given in the ANSWER study, while only $40 \mathrm{~g}$ every 15 days were given in the MACHT trial. Consistent with this reasoning, a prospective non-randomised study showed that the long-term administration of $20 \mathrm{~g}$ of HA twice weekly in patients with cirrhosis and refractory ascites reduced mortality and incidence of complications with respect to SMT. ${ }^{3}$

The different HA doses administered in the ANSWER and MACHT studies probably explain why a significant increase in serum albumin levels (serum albumin) was obtained in the former, while no changes occurred in the latter. That different doses of HA lead to different effects on serum albumin is not only intuitive, but it has also been demonstrated recently. In fact, the weekly administration of $1.5 \mathrm{~g} / \mathrm{kg}$ of body weight of HA induced a greater increase and a greater normalisation rate of serum albumin compared to the infusion of a lower HA dose ( $1 \mathrm{~g} / \mathrm{kg}$ every 2 weeks). ${ }^{4}$ Interestingly, cardiocirculatory dysfunction and systemic inflammation only improved in patients who normalised serum albumin, up to a median close to $4 \mathrm{~g} / \mathrm{dl}^{4}$

Overall, these findings suggest that the amount of circulating albumin achieved by HA administration is relevant in inducing treatment effects. Thus, in the present post hoc analysis of the ANSWER study, we aimed to assess whether on-treatment serum albumin level is associated with survival and incidence of complications. If so, the identification of an on-treatment serum albumin level associated with optimal clinical outcomes could guide long-term HA administration. Moreover, we assessed whether or not albumin administration provides a survival benefit compared to SMT in patients whose ontreatment serum album fails to reach normal levels ( $\geq 3.5 \mathrm{~g} / \mathrm{dl}$ ).

\section{Materials and methods Study design}

This study was a post hoc analysis of the human Albumin for the treatmeNt of aScites in patients With hEpatic ciRrhosis (ANSWER) study. Details of the rationale, study design, characteristics of the participants, and principal results of the ANSWER study have been published elsewhere. ${ }^{1}$ Briefly, the ANSWER study was an investigator-initiated multicentre randomised, parallel, openlabel, pragmatic trial carried out in 33 academic and nonacademic Italian hospitals. Study protocol, amendments, and the informed consent process were first approved by the ethics committee at the coordinating centre (University of Bologna, S. Orsola-Malpighi Hospital, Bologna, Italy) and then at each participating centre. The study was registered with EudraCT, number 2008-000625-19, and ClinicalTrials.gov, number NCT01288794.

In this post hoc analysis, the preliminary and exploratory analyses and the assessment of the association between baseline serum albumin and 18-month mortality risk were based on the entire modified intention-to-treat population from the ANSWER study, that is the population resulting from the exclusion of the patients withdrawn because of wrong inclusion or consent withdrawal. $^{1}$

To assess the association between on-treatment serum albumin level and clinical outcomes, we considered serum albumin determined at month 1 of treatment for 2 reasons: first, in the ANSWER trial, a significant serum albumin increase was reached at that time, remaining substantially steady thereafter (Fig. S1); second, the availability of a good prognostic marker after only 1 month of therapy would have obvious practical implications, including the theoretical possibility of adjusting therapy according to the on-treatment serum albumin level achieved. Due to this choice, the analysis was carried out in patients from the albumin arm of the ANSWER study who were alive with an available serum albumin determination at month 1 , and consisted in counting survival and complications from 1 month up to 18 months after randomisation.

\section{Participants}

Patients aged $\geq 18$ years with cirrhosis and persistent uncomplicated ascites despite ongoing diuretic treatment (at least antialdosteronic drug at a dose $\geq 200 \mathrm{mg} /$ day and furosemide $\geq 25$ $\mathrm{mg}$ /day) were included. The main exclusion criteria included refractory ascites, recent complications of cirrhosis, transjugular intrahepatic portosystemic shunt (TIPS), active hepatocellular carcinoma, liver transplantation, ongoing alcohol abuse, extrahepatic organ failure, and albumin use for the treatment of ascites in the month preceding enrolment. Written informed consent was obtained from all patients.

\section{Procedures}

After enrolment, patients were randomised to receive either SMT or SMT plus HA (20\% HA in $50 \mathrm{ml}$ vials) at a dose of $40 \mathrm{~g}$ twice a week for the initial 2 weeks, and $40 \mathrm{~g}$ weekly thereafter. Patients were assessed monthly for up to 18 months or study interruption or death. At each visit, clinical, laboratory, and instrumental data (if needed) were collected by the attending physicians. The study was interrupted when patients underwent liver transplantation or TIPS insertion, needed $\geq 3$ therapeutic paracenteses per month, refused to continue their participation in the study, or because of medical judgment. Data were recorded on a web-based case report form. More details of the study procedures have been published elsewhere. ${ }^{1}$

\section{Outcomes}

The primary endpoint of the ANSWER trial was 18-month overall mortality. ${ }^{1}$ Among the secondary end-points of the original study, the following were included in this post hoc 
analysis: i) cumulative incidence of paracenteses, and ii) incidence of cirrhosis-related complications (refractory ascites, spontaneous bacterial peritonitis [SBP], other bacterial infections, renal impairment [episodes with serum creatinine concentration $>1.5 \mathrm{mg} / \mathrm{dl}$ ], hepatorenal syndrome type 1 [HRS], hepatic encephalopathy grade 3 or 4 , hyponatraemia [serum sodium concentration $<130 \mathrm{mmol} / \mathrm{L}$ ] or hyperkalaemia [serum potassium concentration $\geq 5.5 \mathrm{mmol} / \mathrm{L}]$ and gastrointestinal bleeding related to portal hypertension).

\section{Statistical analysis}

For quantitative variables mean and SD were reported when the normality of distribution hypothesis was not rejected, otherwise, median and IQR were presented.

In the various steps of our data analysis, the following statistical methods were employed: i) relationship between baseline serum albumin and 18-month risk of death: Cox proportional hazard regression model; ii) identification of ontreatment factors associated with clinical outcomes: univariate and multivariable Cox proportional hazard regression models; in the multivariable model, a backward selection of variables based on the Akaike information criterion was applied for regressor selection. As on-treatment serum albumin was pre-defined at month 1, all other variables entered into the models had been assessed at month 1 ; iii) search for on-treatment (1-month) serum albumin levels able to discriminate patient populations with different probabilities of 18-month survival: Kaplan-Meier estimates. Drawing of the relationship between on-treatment serum albumin and survival: $2^{\text {nd }}$ order polynomial regression; iv) identification of the optimal on-treatment serum albumin level that best discriminates patient populations with different probabilities of 18-month survival: lower $p$ value of KaplanMeier estimates in the serum albumin range above the lower normal limit ( $\geq 3.5 \mathrm{~g} / \mathrm{dl}$ ); v) once the optimal on-treatment serum albumin level was identified, estimate of the risk of bias deriving from a partially data-driven variable: internal 10 -fold cross validation analysis. Further details are reported in Table S3; vi) selection of patients undergoing SMT matched with patients who received albumin administration without normalising ontreatment serum albumin: the probability of failing to reach the threshold of $3.5 \mathrm{~g} / \mathrm{dl}$ at 1 month (principal strata) was considered as a function of basal characteristics and determined through a multivariable logistic regression model. Therefore, the principal score for each individual was determined. ${ }^{5,6}$ We then proceeded to a nearest neighbour principal score matching (1:1 ratio) between the SMT+HA patients (who actually belong to the low on-treatment serum albumin strata) with the SMT group patients showing the closest principal score; vii) probability of 18-month survival in the 2 patient populations selected as above: Kaplan-Meier estimates; viii) cost-effectiveness of longterm albumin administration in the 2 patient populations selected as above: cost-effectiveness analysis as reported in detail elsewhere, using the same cost-drivers. ${ }^{1}$ In summary, costeffectiveness was evaluated by calculating quality-adjusted lifeyears (QALY) from the EQ-5 D utility index and the incremental cost-effectiveness ratio (ICER). ICER values below $€ 35,000 / \mathrm{Q} A \mathrm{LY}$ were considered to indicate cost-effectiveness. ${ }^{7}$ ix) assessment of secondary outcomes: incidence rates (95\% CI) and incidence rate ratios using the exact method on the basis of the Poisson distribution.
All reported $p$ values are 2 -sided and values $<0.05$ were considered statistically significant. All confidence intervals are at the 95\% level. Data were managed with PL/SQL Developer, version 9.0.6.1665 (Allround automation, Enschede, Netherlands) and analyses were carried out by the non-profit Interuniversity Consortium for data collection, data processing, and statistical analysis (CINECA, Bologna, Italy) using R open-source statistical software, version 3.3.1.

\section{Results}

\section{Baseline serum albumin}

The modified intention-to-treat patient population included in the ANSWER study consisted of 213 individuals assigned to the SMT arm and 218 to the SMT+HA arm. ${ }^{1}$ Baseline serum albumin was determined in all cases and did not differ between the 2 arms: SMT $3.10 \pm 0.49 \mathrm{~g} / \mathrm{dl} ;$ SMT+HA $3.09 \pm 0.55 \mathrm{~g} / \mathrm{dl}(p=0.86) .{ }^{1}$ The prevalence of patients with hypoalbuminaemia (serum albumin $<3.5 \mathrm{~g} / \mathrm{dl}$ ) was also similar in the 2 arms (SMT 161 [75.6\%]; SMT+HA 160 [73.4\%]; $p=0.60$ ).

As expected, baseline serum albumin in patients receiving SMT was closely associated with 18-month mortality, with a progressive increase in the risk of death for any $0.1 \mathrm{~g} / \mathrm{dl}$ decrease (hazard ratio [HR] 4.42; 95\% CI 2.34-8.33; $p<0.001$ ). Such a highly significant relationship was effaced in the SMT+HA arm, with a more than halved risk of death for any $0.1 \mathrm{~g} / \mathrm{dl}$ decrease (HR 1.85; 95\% CI 0.99-3.49; $p=0.055$ ).

\section{Effects of long-term albumin administration on serum albumin}

As reported in the ANSWER study publication, ${ }^{1}$ serum albumin level increased significantly within a month in the SMT+HA arm, remaining steady thereafter, while no changes were seen in the SMT arm (Fig. S1). In the SMT+HA arm, 205 patients out of 218 were still followed-up at 1-month. The reasons for study interruption by 13 patients in the first month of follow-up are reported in Table S1. In this population, serum albumin level at 1-month was available in 193 cases: baseline serum albumin was $3.08 \pm 0.54 \mathrm{~g} / \mathrm{dl}$ and rose to $3.83 \pm 0.60 \mathrm{~g} / \mathrm{dl}$ after a month $(p<0.001)$. Such an increase, with a median delta value of $0.75 \mathrm{~g} / \mathrm{dl}$ (IQR $0.4-1.07 \mathrm{~g} / \mathrm{dl}$ ), occurred in $94.3 \%$ of patients, which fits with the 1-month compliance to treatment (97.3\%). Normal serum albumin $(\geq 3.5 \mathrm{~g} / \mathrm{dl})$ at 1 -month of treatment was documented in 153 patients (79.3\%).

\section{Relationship between 1-month on-treatment serum albumin and clinical outcomes}

The factors associated with 18-month mortality were first assessed. Cox regression analysis identified the following variables: age, serum albumin, serum sodium, serum bilirubin, international normalised ratio (INR), Child-Pugh score, model for end-stage liver disease (MELD) score and MELD-Na score (Table 1). Age, serum albumin, Child-Pugh score and MELD-Na score were then entered into the Cox multivariable model: serum albumin and MELD-Na emerged as the only independent predictors (Table 1 ).

It was then assessed whether there are cut-off concentrations of 1-month on-treatment serum albumin that could discriminate patient populations with different probabilities of 18-month survival. Kaplan-Meier estimation showed that this was the case for any $0.1 \mathrm{~g} / \mathrm{dl}$ interval of serum albumin, with a statistical 
Table 1. Cox regression analysis of 1-month factors associated with 18-month mortality.

\begin{tabular}{|c|c|c|c|}
\hline Univariable model & HR & $\mathbf{9 5 \%} \mathrm{CI}$ & $p$ value \\
\hline Female sex (yes $v s$. no) & 0.748 & $(0.372-1.503)$ & 0.414 \\
\hline Age (5 years increase) & 1.230 & $(1.041-1.453)$ & 0.015 \\
\hline Viral aetiology (yes vs. no) & 1.799 & $(0.884-3.658)$ & 0.105 \\
\hline On-treatment serum albumin ( $1 \mathrm{~g} / \mathrm{dl}$ decrease $)$ & 1.129 & $(1.070-1.192)$ & $<0.001$ \\
\hline Serum creatinine ( $1 \mathrm{mg} / \mathrm{dl}$ increase $)$ & 1.427 & $(0.472-4.315)$ & 0.528 \\
\hline Serum potassium ( $1 \mathrm{mmol} / \mathrm{L}$ increase $)$ & 1.115 & $(0.587-2.117)$ & 0.740 \\
\hline Serum sodium (1 mmol/L increase) & 0.868 & $(0.801-0.941)$ & $<0.001$ \\
\hline Serum bilirubin (1 mg/dl increase) & 1.469 & $(1.296-1.666)$ & $<0.001$ \\
\hline International normalised ratio (1-point increase) & 6.242 & $(2.513-15.500)$ & $<0.001$ \\
\hline Haemoglobin ( $1 \mathrm{~g} / \mathrm{dl}$ increase $)$ & 0.921 & $(0.759-1.117)$ & 0.401 \\
\hline Child-Pugh score (1-point increase) & 1.823 & $(1.510-2.201)$ & $<0.001$ \\
\hline MELD score (1-point increase) & 1.263 & $(1.161-1.374)$ & $<0.001$ \\
\hline MELD-Na score (1-point increase) & 1.260 & $(1.164-1.364)$ & $<0.001$ \\
\hline \multicolumn{4}{|l|}{ Multivariable model } \\
\hline On-treatment serum albumin ( $1 \mathrm{~g} / \mathrm{dl}$ decrease $)$ & 1.083 & $(1.021-1.149)$ & 0.008 \\
\hline MELD-Na score (1-point increase) & 1.217 & $(1.120-1.322)$ & $<0.001$ \\
\hline
\end{tabular}

HR, hazard ratio; MELD, model for end-stage liver disease; MELD-Na, MELD score incorporating serum sodium concentration.

Figures in bold refer to statistically significant value.

significance reached for values included in the range $2.5-4.5 \mathrm{~g} / \mathrm{dl}$ (Table S2).

The relationship between 1-month on-treatment serum albumin level and 18-month survival was also analysed with second-order polynomial regression (Fig. 1). The monotonically increasing feature of the curve indicates that survival improves in parallel with serum albumin, even in those patients whose serum albumin increased beyond the lower normal limit. In this patient subgroup, the cut-off value that best discriminated patient survival was $4.0 \mathrm{~g} / \mathrm{dl}$, a value observed in $75(38.9 \%)$ patients (Table S2; Fig. 2). Patients reaching the 1-month ontreatment serum albumin $\geq 4.0 \mathrm{~g} / \mathrm{dl}$ showed a reduction in mortality of $80 \%$ compared to patients who did not achieve this threshold (HR 0.20; 95\% CI 0.08-0.52; $p<0.001$ ). As a different validation sample was not available, an internal cross validation analysis was conducted showing that the risk of optimism deriving from the use of a partially data-driven threshold could be excluded (Table S3).

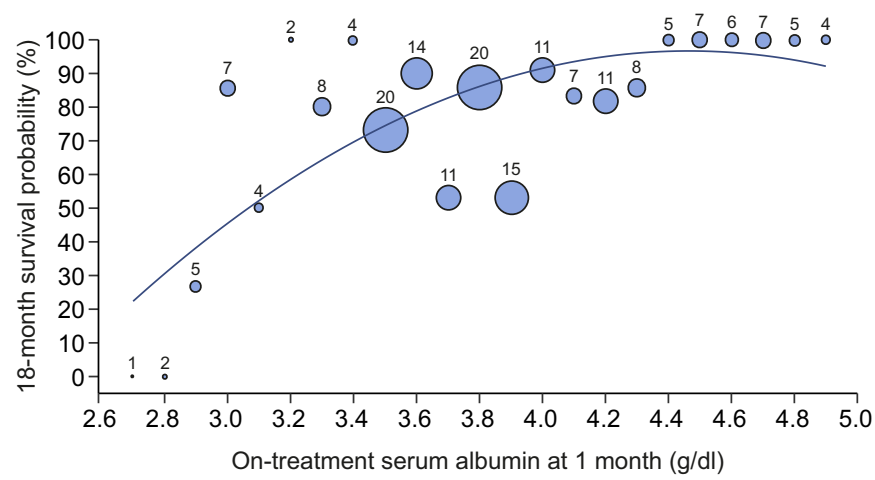

Fig. 1. Relationship of 1-month on-treatment serum albumin and survival Second-order polynomial regression, including 95\% of observations (185 patients), shows a close relationship between patient 18-month overall survival and on-treatment serum albumin concentration at month 1 (intercept $=-23.87$, $p=0.005)$. The area of each bubble reflects the number of patients for each ontreatment serum albumin level. The position of the bubbles is determined by the survival estimation with Kaplan-Meier method for each given ontreatment serum albumin level. The monotonically increasing feature of the curve indicates that 18-month survival improves in parallel with on-treatment serum albumin concentration, even in those patients who reached serum albumin levels within the normal range ( $\geq 3.5 \mathrm{~g} / \mathrm{dl}$ ).
The same serum albumin cut-off was also excellent in discriminating populations with a different risk of refractory ascites (HR 0.37; 95\% CI 0.18-0.75; $p=0.006$ ) and different cumulative incidences of therapeutic paracentesis and complications of cirrhosis such as bacterial infections (SBP and non-SBP), renal impairment, hepatic encephalopathy grade III and IV, hyponatraemia and hyperkalaemia (Fig. 3). As in the

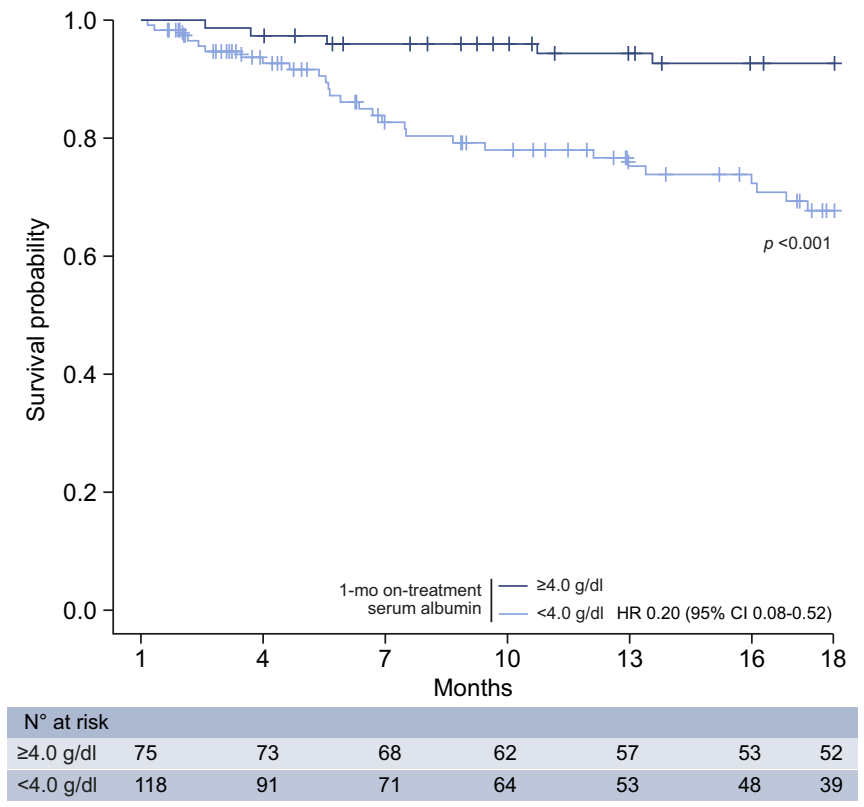

Fig. 2. On-treatment (1-month) serum albumin $4.0 \mathrm{~g} / \mathrm{dl}$ and survival. Kaplan-Meier estimates for the probability of overall 18 -month survival in patients receiving long-term albumin administration who reached (blue) or not (light blue) the on-treatment serum albumin concentration of $4.0 \mathrm{~g} / \mathrm{dl}$. The $p$ value was calculated with the log-rank test. The reasons for censoring in patients whose on-treatment serum albumin was $<4 \mathrm{~g} / \mathrm{dl}$ were: liver transplantation (13 cases), refractory ascites requiring $\geq 3$ paracenteses in a month (12 cases), patient decision (14 cases), lost to follow-up (6 cases), TIPS insertion for refractory ascites (3 cases), TIPS insertion for oesophageal bleeding, physician decision and protocol violation ( 1 case for each reason). The reasons for censoring in patients whose on-treatment serum albumin was $\geq 4 \mathrm{~g} / \mathrm{dl}$ were: liver transplantation ( 3 cases), refractory ascites requiring $\geq 3$ paracenteses in a month (5 cases), patient decision ( 4 cases), lost to follow-up ( 4 cases) and protocol violation (2 cases). 


\section{JOURNAL OF HEPATOLOGY}

original study, most adverse events were related to cirrhosis. The frequency of unanticipated adverse events and adverse reactions potentially related to HA administration did not differ between patients with on-treatment serum albumin $<$ or $\geq 4.0 \mathrm{~g} / \mathrm{dl}$ (Table S4A,B).

Univariate logistic regression identified baseline serum albumin, serum bilirubin, INR, and Child-Pugh, MELD and MELD-Na scores as predictors of the probability of reaching the 1-month on-treatment serum albumin value of $4.0 \mathrm{~g} / \mathrm{dl}$ (Table 2). The following multivariable model showed that the sole independent predictors were baseline serum albumin and MELD score. Indeed, the probability of reaching this threshold lowered for any $0.1 \mathrm{~g} / \mathrm{dl}$ of serum albumin decrease and for any 1-point MELD score increase (Table 2).

Finally, in order to assess whether long-term albumin administration was in any way beneficial in the 40 patients whose on-treatment serum albumin level did not reach the lower normal limit, their survival was compared with that of 40 matched patients enrolled in the SMT who were alive at month 1. To do so, a principal score was built from variables identified by a multivariable analysis (Table S5; Fig. S2).

In this patient population (Table S6), 10 died in the SMT+HA arm and 17 in the SMT arm. As a result, the 40 patients who received HA had a significantly higher 18 -month probability of overall survival (Kaplan-Meier estimates: 61.3\% SMT+HA vs. $36.7 \%$ SMT; $p=0.032$; Fig. 4), corresponding to a $57 \%$ reduction in mortality HR $(0.43 ; 95 \% \mathrm{CI} 0.19-0.95 ; p=0.036)$. The 40 patients in the SMT+HA arm also showed a significant reduction in the cumulative incidence of some complications of cirrhosis, namely hepatic encephalopathy grade III or IV and hyponatraemia (Fig. 5). At last, cost-effective analysis showed that the ICER calculated in patients who received long-term albumin administration was $€ 108.58 /$ patient/year. As the incremental QALY gain was $0.101 /$ year, the ICER/QALY ratio was $€ 1,074.95$ (Table S7).

\section{Discussion}

This post hoc analysis of the ANSWER study, a multicentre, randomised, non-profit, open-label clinical trial assessing the effects of long-term albumin administration to patients with decompensated cirrhosis, ${ }^{1}$ provided 6 interesting findings most of which could be relevant for clinical practice. First, long-term albumin administration almost completely effaced the wellknown relationship between hypoalbuminaemia and patient mortality. Second, after 1 month of treatment, serum albumin was the sole independent predictor of survival along with MELD-Na score. Third, a parallel increase between 1-month ontreatment serum albumin and survival was found, even in those patients presenting with serum albumin within the normal range. Fourth, the on-treatment serum albumin cut-off value that best discriminated patient survival was $4.0 \mathrm{~g} / \mathrm{dl}$, a level that also well differentiated patients in terms of occurrence of major complications of cirrhosis. Fifth, the baseline independent predictors of reaching this threshold were serum albumin and MELD score. Sixth, even those patients who failed to increase 1-month on-treatment serum albumin levels up to the normal range received a benefit from long-term albumin administration in
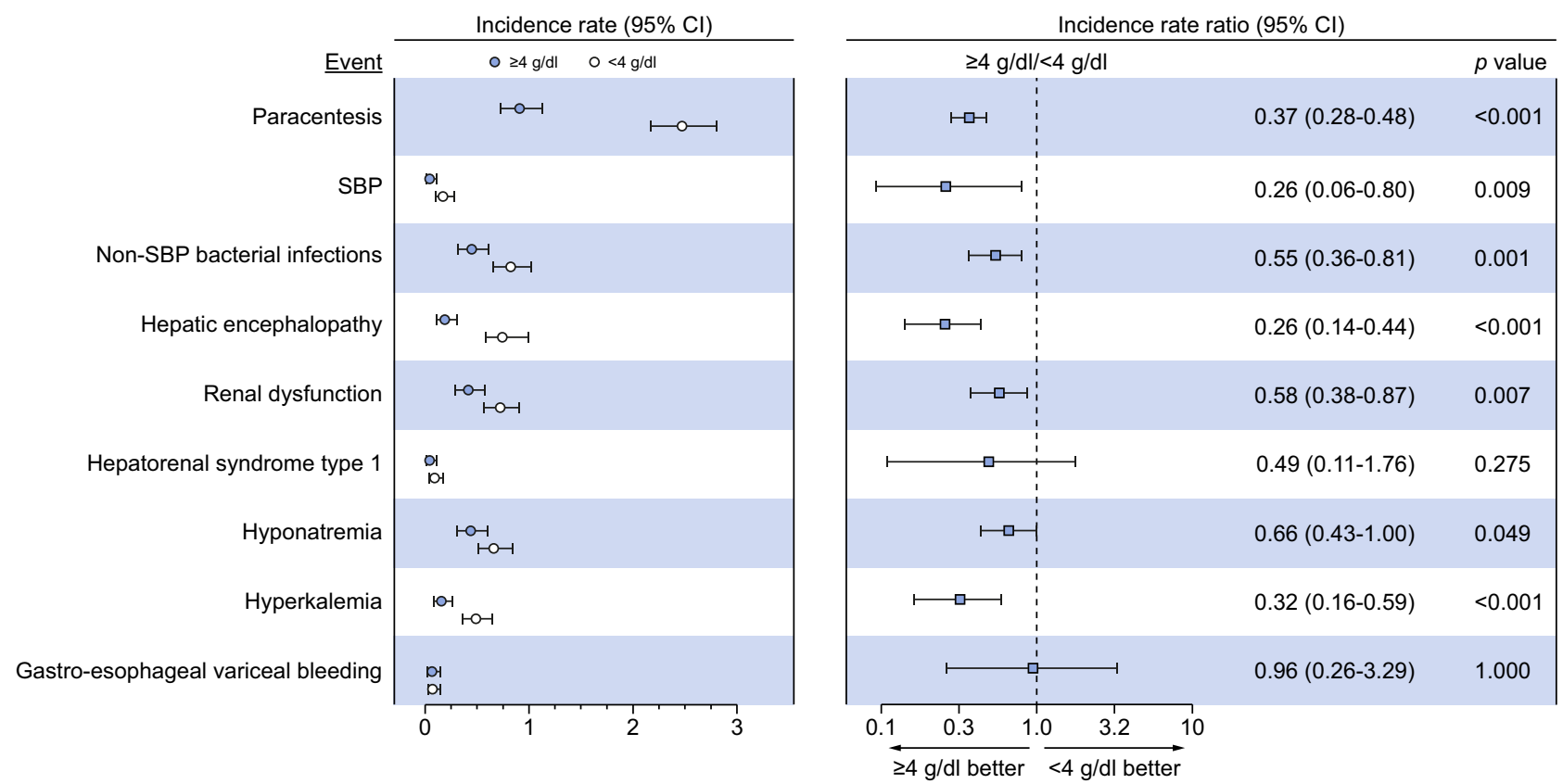

Fig. 3. On-treatment (1-month) serum albumin of $\mathbf{4 . 0} \mathbf{~ g / d l}$ and complications of cirrhosis. The figure shows the incidence of complications of cirrhosis in patients receiving long-term albumin administration who reached or not the on-treatment serum albumin of $4.0 \mathrm{~g} / \mathrm{dl}$. Left panel: incidence rate, expressed as number of events per person per year (bars report 95\% CI), of therapeutic paracentesis and complications of cirrhosis in patients who reached (black dots) or not (white dots) the on-treatment serum albumin concentration of $4.0 \mathrm{~g} / \mathrm{dl}$ (black dots). Right panel: Forrest plot of the incidence rate ratios of therapeutic paracentesis and complications in patients who reached or not the on-treatment serum albumin concentration of $4.0 \mathrm{~g} / \mathrm{dl}$. Incidence rate ratios (with $95 \% \mathrm{CI}$ ) $<1$ indicates a statistically significant reduction. SBP = episodes of spontaneous bacterial peritonitis. Non-SBP bacterial infections $=$ episodes of bacterial infections other than SBP. Hepatic encephalopathy = episodes of hepatic encephalopathy grade III and IV. Renal dysfunction = episodes with serum creatinine concentration above $1.5 \mathrm{mg} / \mathrm{dl}$. Hepatorenal syndrome type 1 = episodes of hepatorenal syndrome type 1 . Hyponatraemia = episodes of serum sodium concentration <130 $\mathrm{mmol} / \mathrm{L}$. Hyperkalaemia $=$ episodes of serum potassium concentration $\geq 5.5 \mathrm{mmol} / \mathrm{L}$. Gastro-oesophageal variceal bleeding $=$ episodes of bleeding due to rupture of oesophageal or gastric varices. 
Table 2. Baseline predictors of the probability of reaching the on-treatment serum albumin value of $4.0 \mathrm{~g} / \mathrm{dl}$ in the SMT plus HA arm.

\begin{tabular}{|c|c|c|c|}
\hline Univariable model & OR & $95 \% \mathrm{CI}$ & $p$ value \\
\hline Female sex (yes $v s$. no) & 1.173 & $(0.621-2.217)$ & 0.623 \\
\hline Age (5 years increase) & 1.012 & $(0.890-1.150)$ & 0.856 \\
\hline Viral aetiology (yes vs. no) & 0.892 & $(0.494-1.626)$ & 0.710 \\
\hline Comorbidity (yes vs. no) & 1.284 & $(0.701-2.351)$ & 0.418 \\
\hline Mean arterial pressure ( $1 \mathrm{mmHg}$ increase $)$ & 0.994 & $(0.962-1.027)$ & 0.726 \\
\hline Heart rate (1-beat per minute increase) & 0.991 & $(0.964-1.018)$ & 0.510 \\
\hline Baseline serum albumin (0.1 $\mathrm{g} / \mathrm{dl}$ increase) & 1.171 & $(1.094-1.253)$ & $<0.001$ \\
\hline Serum creatinine ( $1 \mathrm{mg} / \mathrm{dl}$ increase $)$ & 1.765 & $(0.509-6.116)$ & 0.370 \\
\hline Serum potassium ( $1 \mathrm{mmol} / \mathrm{L}$ increase $)$ & 0.917 & $(0.529-1.589)$ & 0.757 \\
\hline Serum sodium ( $1 \mathrm{mmol} / \mathrm{L}$ increase $)$ & 1.034 & $(0.959-1.115)$ & 0.383 \\
\hline Hyponatraemia $^{\#}$ (yes vs. no) & 0.735 & $(0.384-1.404)$ & 0.351 \\
\hline Serum bilirubin ( $1 \mathrm{mg} / \mathrm{dl}$ increase $)$ & 0.725 & $(0.585-0.898)$ & 0.003 \\
\hline International normalised ratio (1-unit increase) & 0.170 & $(0.049-0.797)$ & 0.023 \\
\hline Haemoglobin ( $1 \mathrm{~g} / \mathrm{dl}$ increase) & 1.145 & $(0.970-1.350)$ & $0.10 \mathrm{~s}$ \\
\hline Platelets ( $50 \times 10^{3} / \mathrm{mmc}$ increase) & 1.043 & $(0.846-1.287)$ & 0.693 \\
\hline White blood cell count $\left(10^{3} / \mathrm{mmc}\right.$ increase $)$ & 1.040 & $(0.902-1.199)$ & 0.590 \\
\hline Baseline Child-Pugh score (1-point increase) & 0.600 & $(0.471-0.763)$ & $<0.001$ \\
\hline Baseline MELD score (1-point increase) & 0.854 & $(0.777-0.937)$ & $<0.001$ \\
\hline Baseline MELD-Na score (1-point increase) & 0.906 & $(0.844-0.971)$ & 0.006 \\
\hline Paracentesis* (yes vs. no) & 1.373 & $(0.666-2.829)$ & 0.391 \\
\hline Inpatient at randomisation (yes vs. no) & 1.107 & $(0.540-2.271)$ & 0.782 \\
\hline \multicolumn{4}{|l|}{ Multivariable model } \\
\hline Baseline serum albumin (0.1 g/dl increase) & 1.147 & $(1.069-1.229)$ & $<0.001$ \\
\hline Baseline MELD score (1-point increase) & 0.903 & $(0.820-0.995)$ & 0.040 \\
\hline
\end{tabular}

MELD, model for end-stage liver disease; MELD-Na, MELD score incorporating serum sodium concentration; OR, odds ratio.

Figures in bold refer to statistically significant value.

${ }^{*}$ Paracentesis within a month before randomisation.

${ }^{*}$ Hyponatraemia was defined as serum sodium level $\leq 135 \mathrm{mmol} / \mathrm{L}$.

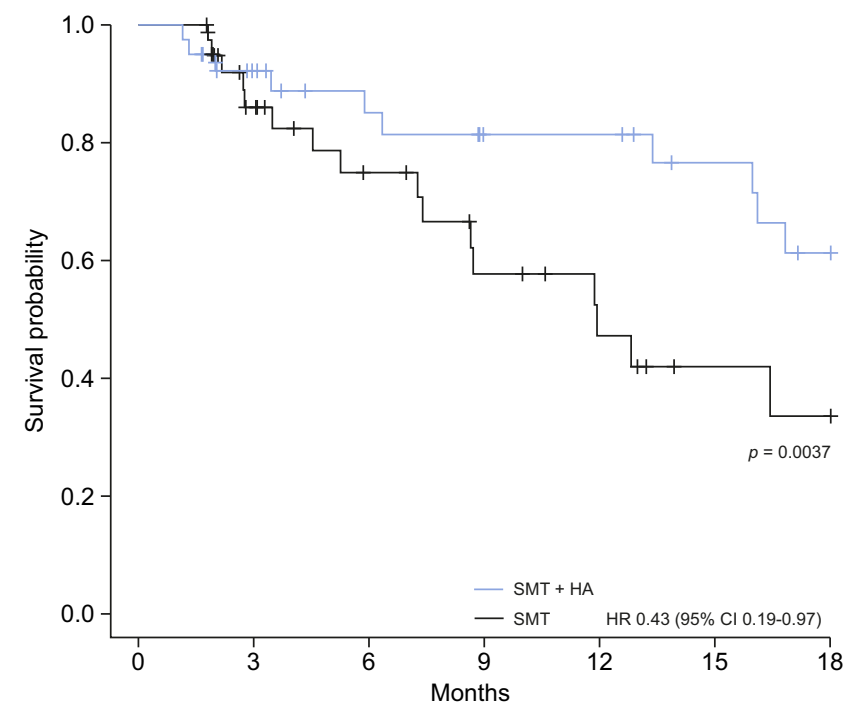

$\begin{array}{llllllll}\mathrm{N}^{\circ} \text { at risk } & & & & & & & \\ \mathrm{SMT}+\mathrm{HA} & 40 & 29 & 23 & 19 & 19 & 15 & 11 \\ \mathrm{SMT} & 40 & 27 & 19 & 13 & 9 & 5 & 4\end{array}$

Fig. 4. Survival of patients failing to normalise serum albumin at 1 month. Fig. shows Kaplan-Meier estimates for the probability of overall 18-month survival in patients divided according to treatment groups. The blue line represents the 40 patients whose on-treatment serum albumin concentration did not reach the lower normal limit $(3.5 \mathrm{~g} / \mathrm{dl})$. The grey line represents the 40 untreated patients matched with principal score technique. The $p$ value was calculated with the log-rank test. The reasons for censoring in patients who received standard medical treatment were: liver transplantation (6 cases) refractory ascites requiring $\geq 3$ paracenteses in a month (6 cases), patient decision (4 cases), lost to follow-up, TIPS insertion for oesophageal bleeding and protocol violation (1 case for each reason). The reasons for censoring in patients who received standard medical treatment plus albumin were: liver transplantation (8 cases), refractory ascites requiring $\geq 3$ paracenteses in a month ( 4 cases), patient decision ( 4 cases), TIPS for refractory ascites ( 2 cases) and lost to follow-up ( 1 case). terms of survival and occurrence of complications. In this subset of patients, albumin treatment was also cost-effective.

The adverse prognostic meaning of hypoalbuminaemia in patients with cirrhosis has long been recognised, ${ }^{8}$ so that serum albumin is included in widely used prognostic scores, such as the Child-Pugh score. ${ }^{9}$ Thus, the close inverse association between baseline serum albumin and mortality found in patients randomised to the SMT was largely expected. Instead, what appears a new finding is that this relationship was almost effaced in patients treated with albumin. This result implies that baseline serum albumin per se should not guide the decision to start albumin administration. Rather, the indication for this type of treatment should stem from a comprehensive clinical appraisal, as was the case in the ANSWER study that enrolled patients with persistent ascites despite a moderate diuretic therapy. ${ }^{1}$

An even more clinically relevant observation would be that, to obtain favourable results, long-term albumin administration should induce an increase in serum albumin. In order to reinforce this assumption, it should be assessed whether a relationship between on-treatment serum albumin and outcomes exists. To do so, a given on-treatment serum albumin value should be identified as a reference. As reported in the study design, 1-month on-treatment serum albumin level was used for this purpose.

Several findings in this study support the choice of employing 1-month serum albumin as an on-treatment reference value. Besides MELD-Na score, serum albumin was the sole independent on-treatment predictor of survival. Such a close association was further confirmed by polynomial regression. The latter analysis also showed that serum albumin and survival increased in parallel, even once serum albumin had reached the normal range, a result achieved by the majority (79\%) of patients. 

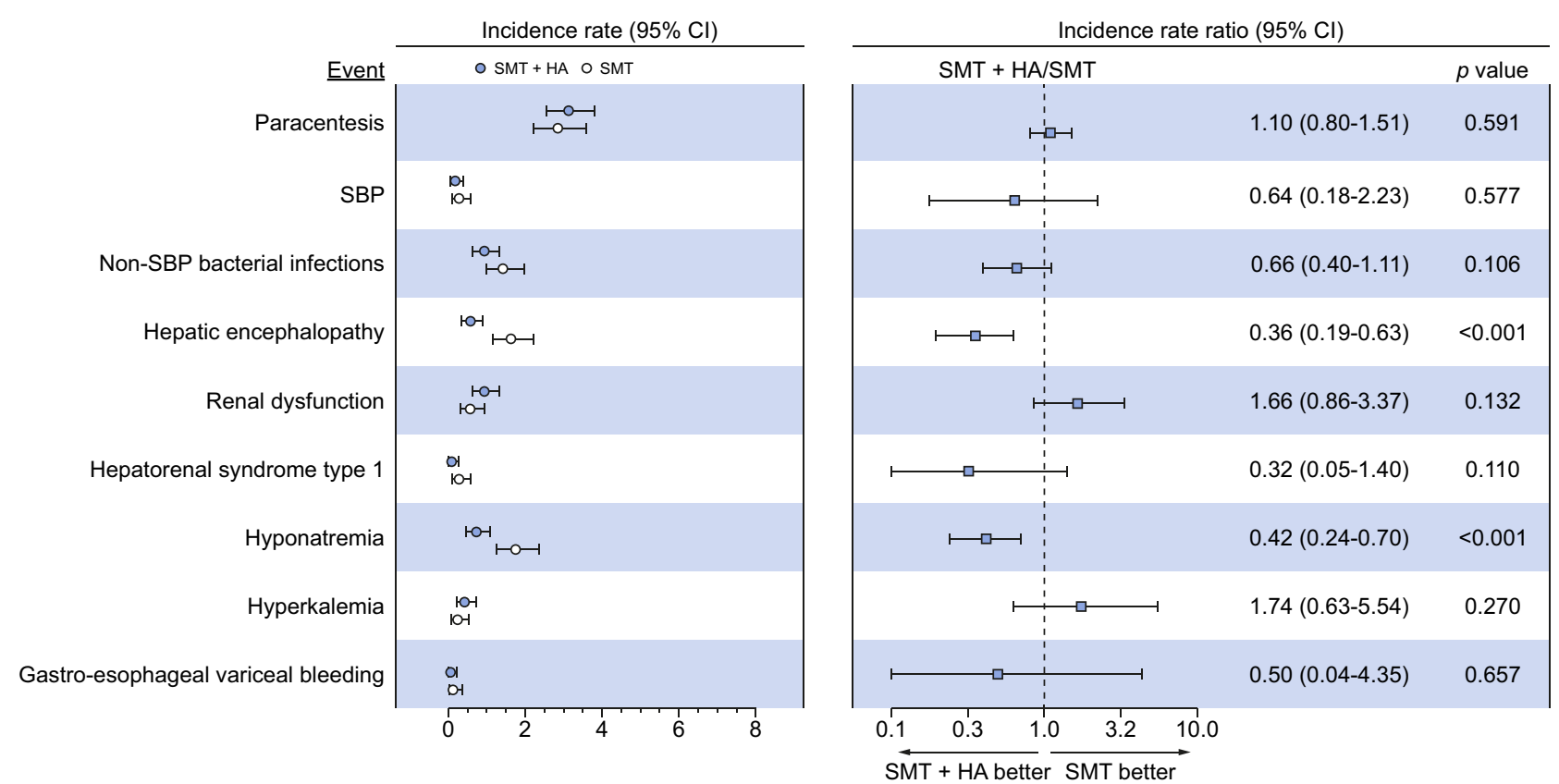

Fig. 5. Complications of cirrhosis in patients failing to normalise serum albumin at 1 month. Incidence rates of complications of cirrhosis in the 40 patients whose on-treatment serum albumin concentration did not reach the lower normal limit $(3.5 \mathrm{~g} / \mathrm{dl})$ and in the 40 untreated patients matched with principal score technique. Left panel: incidence rate, expressed as number of events per person per year (bars report $95 \% \mathrm{CI}$ ), of therapeutic paracentesis and complications of cirrhosis in the 40 patients who did not reach the on-treatment serum albumin concentration of $3.5 \mathrm{~g} / \mathrm{dl}$ (lower normal limit) (black dots) and in the 40 intreated patients matched with principal score technique (white dots). Right panel: Forrest plot of the incidence rate ratio of therapeutic paracentesis and complications in the 40 patients who did not reach on-treatment serum albumin of $3.5 \mathrm{~g} / \mathrm{dl}$ and in the 40 untreated patients matched with principal score technique. Incidence rate ratios (with $95 \% \mathrm{CI}$ ) $<1$ indicates a statistically significant reduction. SBP = episodes of spontaneous bacterial peritonitis. Non-SBP bacterial infections = episodes of bacterial infections other than SBP. Hepatic encephalopathy $=$ episodes of hepatic encephalopathy grade III and IV. Renal dysfunction $=$ episodes with serum creatinine concentration above $1.5 \mathrm{mg} / \mathrm{dl}$. Hepatorenal syndrome type $1=$ episodes of hepatorenal syndrome type 1 . Hyponatraemia = episodes of serum sodium concentration $<130 \mathrm{mmol} / \mathrm{L}$. Hyperkalaemia = episodes of serum potassium concentration $\geq 5.5 \mathrm{mmol} / \mathrm{L}$. Gastro-oesophageal variceal bleeding $=$ episodes of bleeding due to rupture of oesophageal or gastric varices.

These findings suggest that albumin administration should aim at ensuring the highest possible serum albumin level, as a further survival advantage is associated with pushing serum albumin levels above the lower normal limit. This concept gives rise to the idea of modulating the albumin dose during longterm albumin administration according to patient characteristics. This reasoning led us to look for a potential on-treatment serum albumin threshold able to discriminate patient populations with different survival rates. By performing KaplanMeier estimates at any $0.1 \mathrm{~g} / \mathrm{dl}$ intervals of serum albumin within the range from $3.5-4.5 \mathrm{~g} / \mathrm{dl}$, it emerged that $4.0 \mathrm{~g} / \mathrm{dl}$ was the best discriminant value (Table S1; Fig. 2). Interestingly, in the pilot-PRECIOSA study, an improvement of cardiocirculatory dysfunction and systemic inflammation in patients with decompensated cirrhosis was only achieved when the ontreatment median serum albumin concentration reached $3.92 \mathrm{~g} / \mathrm{dl}$, a level very close to the threshold identified in the current study. ${ }^{4}$ Furthermore, such a result was achieved with the infusion of high ( $1.5 \mathrm{~g} / \mathrm{kg}$ body weight weekly), but not low $(1 \mathrm{~g} / \mathrm{kg}$ body weight every 2 weeks) doses of albumin. Moreover, the $4.0-4.4 \mathrm{~g} / \mathrm{dl}$ range of serum albumin is the most frequently seen in healthy individuals belonging to the same age range as our patients. ${ }^{10}$

Based on these observations, we propose considering this cutoff as the ideal on-treatment serum albumin target to be reached to ensure optimal outcomes. Such a target was not only the best discriminant for survival, but also provided a good patient distinction concerning the incidence rate of all complications of cirrhosis analysed in the ANSWER study with the sole exception of HRS (Fig. 3). The latter result is likely influenced by the reduced patient sample size with respect to the original study since a trend in favour of the patient group with serum albumin equal to or above $4.0 \mathrm{~g} / \mathrm{dl}$ was clearly evident. Notably, as in the original study, no difference in the incidence rate of portal hypertensive bleeding was found.

Once an optimal on-treatment serum albumin concentration has been identified, it would be of interest to ascertain whether the probability of reaching this target can be predicted before starting treatment. The multivariable Cox regression model showed that the lower baseline serum albumin and the higher baseline MELD score, the lower the probability of reaching this on-treatment threshold (Table 2). Thus, this goal can be ascertained by using very simple and easily obtainable variables.

The observation that incremental levels of on-treatment serum albumin were associated with incremental probabilities of survival raises the question of whether the minority of patients with persistent hypoalbuminaemia despite treatment actually received a benefit from albumin administration. To answer this question, the 40 patients who still showed reduced serum albumin after 1 month of albumin administration were matched with 40 patients identified from the SMT arm with a principal score technique. Survival analysis showed that even in this population the addition of HA to SMT significantly improved 


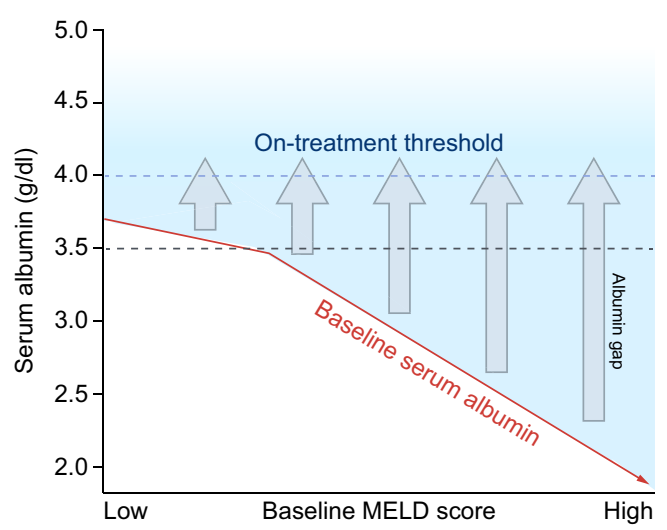

Fig. 6. Graphical representation of the "filling the albumin gap" hypothesis. Long-term albumin treatment of patients with decompensated cirrhosis should be directed at filling the gap existing between baseline serum albumin and a given on-treatment serum albumin target known to be associated with very good outcomes. The results of this study indicated that this target could be fixed at around $4.0 \mathrm{~g} / \mathrm{dl}$. Baseline serum albumin concentration and MELD score were the independent predictors of the probability of reaching such a threshold, thus influencing the amount of albumin to be administered (vertical arrows).

18-month survival, although the survival probability was much lower than that observed in the whole treated cohort in the ANSWER trial (Fig. 4). Furthermore, despite the small sample size, HA administration was associated with a reduction in the incidence of some major complications of cirrhosis such as hepatic encephalopathy grade III or IV and hyponatraemia (Fig. 5). At last, cost-effectiveness analysis showed that long-term albumin administration in this patient subset was associated with a ICER/QALY ratio far below the threshold adopted by National Institute for Health and Care Excellence to consider a treatment cost-effective. ${ }^{7}$ These results imply that this form of treatment cannot be seen as futile in this patient subset. On the contrary, this observation suggests that an increase in the HA dosage, possibly limited to the loading dose period, can be considered to reach serum albumin thresholds associated with better outcomes. In this respect, the predictors of the probability of reaching the optimal on-treatment serum albumin could help in identifying the patients at risk of failing this goal and, therefore, may benefit from a weekly HA dose higher than the fixed amount given in the ANSWER study. The results of the pilotPRECIOSA study suggest that this may be the case. ${ }^{4}$ Increasing the weekly rate of HA infusion may also be useful but would increase the organizational burden.

An issue of great importance is that in patients with cirrhosis the extent of albumin molecule deterioration increases in parallel with the severity of the disease. ${ }^{11}$ This would certainly influence the modalities of HA administration, especially in patients with advanced cirrhosis, but would require the assessment of "effective" albumin levels. ${ }^{12}$ Unfortunately, this is not currently available in clinical practice.

This study presents the limitations of post hoc analyses. ${ }^{13}$ It has to be considered that the heterogeneity of treatment effect, that is how the albumin infusion effects can vary across patients, can only be explored by a post hoc approach. Of course, the inherent risk of low statistical power, multiplicity and false positive and negative results, which we tried to minimise, has to be recognised. However, secondary subgroup analyses to explore more uncertain or unexpected relationships between individual patient attributes and treatment effects are considered appropriate to generate hypotheses, which can then be tested in future studies. ${ }^{14}$ This analysis was conducted on the results of a single randomised clinical trial, the ANSWER study, with the inherent difficulties in generalization and transferability, even though the investigation was a large multicentre study including more than 400 patients. Thus, external validation and calibration of risk prediction are required to translate the results of our analysis into clinical practice. ${ }^{15}$

Globally, the results that emerged from this analysis of the ANSWER trial, as well as the observation derived from the MACHT and pilot-PRECIOSA studies, ${ }^{2,4}$ prompt us to hypothesise that maintaining a serum albumin level of about $4 \mathrm{~g} / \mathrm{dl}$ could optimise clinical outcomes in patients with cirrhosis and persistent ascites. As a result, the goal of longterm albumin administration should be to fill the gap existing between the actual patient serum albumin and the theoretical optimal on-treatment target of serum albumin that we identified. As the probability of filling the gap is variable depending mostly on the starting level of serum albumin and on the severity of cirrhosis, the need emerges to go beyond a fixed dosage and schedule of HA administration - as used in the ANSWER study - to a more individualised treatment adapted to the extent of the gap (Fig. 6). In this context, the time-course changes of serum albumin could be used as a guide to maximise the beneficial effects of the treatment and optimise albumin utilisation. Future studies are certainly needed to validate this hypothesis.

\section{Abbreviations}

HA, human albumin; HR, hazard ratio; HRS, hepatorenal syndrome; ICER, incremental cost-effectiveness ratio; INR, international normalised ratio; MELD, model for end-stage liver disease; QALY, quality-adjusted life-years; SBP, spontaneous bacterial peritonitis; SMT, standard medical treatment; TIPS, transjugular intrahepatic portosystemic shunt.

\section{Financial support}

The ANSWER trial was funded by the competitive grant FARM6P824B from the Italian Medicine Agency.

\section{Conflict of interest}

PC is part of the speakers' bureau for Grifols SA, Octapharma AG, Baxalta, and Kedrion Biopharma, is consultant for Kedrion Biopharma, is on the advisory board for Grifols SA, and has a research grant from Octapharma AG. MT is part of the speakers' bureau for Grifols SA and Octapharma AG. GZ is part of the speakers' bureau for Octapharma. OR is part of speakers' bureau for Baxalta. PA is part of the speakers' bureau for Baxalta and Kedrion Biopharma. PT is part of the speakers' bureau for Grifols and Kedrion Biopharma. MBa is part of the speakers' bureau Octapharma AG. MBe is part of the speakers' bureau for Grifols SA, Octapharma AG, Baxalta, CLS Behring GmbH, and PPTA, and is a consultant for CLS Behring GmbH, Grifols SA and Baxalta. All other authors have no competing interests.

Please refer to the accompanying ICMJE disclosure forms for further details. 


\section{JOURNAL OF HEPATOLOGY}

\section{Authors' contributions}

The original concept of this study was developed by MBe and PC. The study was designed and planned by PC, MT, GZ and MBe. The chief investigators were MBe and PC. Acquisition of the data was done by PC, MT, GZ, OR, PA, CA, SNe, FGF, FL, AAi, LS, GS-B, SF, RGR, RC, VDM, VS., FM, PT, AT, RDM, SN, SP, CE. Statistical analysis was done by AR and MBa. Data analysis and interpretation was done by PC, MBe, MT, GZ, and MBa. The manuscript was drafted by PC, MT and MBe. Images were processed by MT. All authors critically reviewed the manuscript and approved the final draft for submission. The guarantor of the manuscript is MBe.

\section{Data availability statement}

The datasets generated during and/or analysed during the current study are available from the corresponding author on request.

\section{Acknowledgments}

The trial was funded by the competitive peer-reviewed grant FARM6P824B from the Italian Medicine Agency. We thank the Italian Association for the Study of the Liver, the Italian Society of Gastroenterology, and the Italian Association of Hospital Gastroenterologists for their endorsement of this study. The methodological advice from Dr. Agostino Colli is gratefully acknowledged. We also thank the study patients and their relatives for their participation, and the physicians and nursing staff of the participating centres for their cooperation.

\section{ANSWER study investigators}

Marco Domenicali, Ferdinando A Giannone, Agnese Antognoli (Department of Medical and Surgical Sciences, University of Bologna); Manuela Merli, Chiara Pasquale, Stefania Gioia (Department of Clinical Medicine, Sapienza University of Rome); Silvano Fasolato, Antonietta Sticca (Unit of Internal Medicine and Hepatology, Department of Medicine, University of Padua); Daniela Campion, Alessandro Risso, Giorgio M Saracco (Division of Gastroenterology and Hepatology, Città della Salute e della Scienza Hospital, University of Turin); Loredana Prestianni, Federica Fidone, Daniela Maiorca, Agostino Rizzotto (Department of Clinical and Experimental Medicine, University of Catania); Federica Mirici Cappa, Arianna Lanzi, Elga Neri, Anna Visani (Internal Medicine, Hospital of Faenza, AUSL of Romagna); Antonio Mastroianni (Internal Medicine, Hospital of Bentivoglio, AUSL of Bologna); Giovanni Perricone, Alberto B Alberti, Lucia Cesarini, Chiara Mazzarelli, Marcello Vangeli, Raffaella Viganò (Liver Unit, Department of Hepatology and Gastroenterology, Niguarda Hospital, Milan); Marco Marzioni, Francesca Capretti, Alba Kostandini (Department of Gastroenterology, PolItechnic University of Marche, Ancona); Giulia Magini, Maria Colpani (Gastroenterology and Transplant Hepatology, Papa Giovanni XXIII Hospital, Bergamo); Tommaso Gabbani, Maria Marsico (Department of Experimental and Clinical Medicine, University of Florence); Marianna Zappimbulso, Josè Petruzzi (Division of Gastroenterology, National Institute of Gastroenterology S De Bellis, Castellana Grotte); Vincenza Calvaruso (Unit of Gastroenterology and Hepatology, Biomedical Department of Internal and Specialistic Medicine, University of Palermo); Giovanni Parrella (AORN dei Colli "Cotugno" Hospital of Naples); Nicola Caporaso, Francesco Auriemma, Maria Guarino (Department of Clinical Medicine and
Surgery, Gastroenterology Unit, Federico II University of Naples); Fabio Pugliese (Internal Medicine, Department of Medical Area, University of Udine); Annalisa Tortora (Gastroenterology, Gemelli Foundation, Cattolica University, Rome); Pietro Leo (Gastroenterology Unit, Hospital of Cosenza); Mario Angelico, Francesco De Leonardis, Alessandra Pecchioli, Piera Rossi (Liver Unit, Tor Vergata University, Rome); Giovanni Raimondo, Irene Cacciola (Division of Clinical and Molecular Hepatology, University Hospital of Messina); Gianfranco Elia, Elisa Negri (Infectious Diseases and Hepatology, University Hospital of Parma, Italy); Marcello Dallio, Carmelina Loguercio, Alessandro Federico (University of Campania Luigi Vanvitelli, Naples); Dario Conte, Sara Massironi (Gastroenterology and Endoscopy Unit, Foundation IRCSS Ca' Granda Ospedale Maggiore Policlinico, Milan); Giorgio Ballardini Natascia Celli (AUSL of Romagna, Internal Medicine, Hospital of Rimini); Maria Rendina, Roberto Bringiotti, Nicola Maurizio Castellaneta (Section of Gastroenterology, Department of Emergency and Organ Transplantation, University of Bari); Francesco Salerno (Internal Medicine, IRCSS Policlinico San Donato, University of Milan); Sergio Boccia (Gastroenterology Unit, University Hospital, Ferrara); Riccardo Guarisco (Internal Medicine, S Sebastiano General Hospital, Frascati); Alessandra Galioto (Internal Medicine, Hospital of Dolo, Azienda Unità Locale Socio-sanitaria Serenissima, Mestre); Marta Cavallin (Unit of Internal Medicine and Hepatology, Department of Medicine, University of Padua); Alida Andrealli (Division of Gastroenterology and Hepatology, Città della Salute e della Scienza Hospital, University of Turin).

\section{Supplementary data}

Supplementary data associated with this article can be found, in the online version, at https://doi.org/10.1016/j.jhep.2020.08.021.

\section{References}

Author names in bold designate shared co-first authorship

[1] Caraceni P, Riggio O, Angeli P, Alessandria C, Neri S, Foschi FG, et al. Longterm albumin administration in decompensated cirrhosis (ANSWER): an open-label randomised trial. Lancet 2018;391:2417-2429.

[2] Solá E, Solé C, Simón-Talero M, Martín-Llahí M, Castellote J, GarciaMartínez RR, et al. Midodrine and albumin for prevention of complications in patients with cirrhosis awaiting liver transplantation. A randomized placebo-controlled trial. J Hepatol 2018;69:1250-1259.

[3] Di Pascoli M, Fasolato S, Piano S, Bolognesi M, Angeli P. Long-term administration of human albumin improves survival in patients with cirrhosis and refractory ascites. Liver Int 2019;39:98-105.

[4] Fernàndez J, Clária J, Amorós A, Aguilar F, Castro M, Casulleraset M, et al. Effects of albumin treatment on systemic and portal hemodynamics and systemic inflammation in patients with decompensated cirrhosis. Gastroenterology 2019;157:149-162.

[5] Stuart EA, Jo B. Assessing the sensitivity of methods for estimating principal causal effects. Stat Methods Med Res 2015;24:657-674.

[6] Porcher R, Leyrat C, Baron G, Giraudeau B, Boutron I. Performance of principal scores to estimate the marginal compliers causal effect of an intervention. Stat Med 2016;35:752-767.

[7] McCabe C, Claxton K, Culyer AJ. The NICE cost-effectiveness threshold: what it is and what that means. Pharmacoeconomics 2008;26:733744.

[8] Salerno F, Borroni G, Moser P, Badalamenti S, Cassarà L, Maggi A, et al Survival and prognostic factors of cirrhotic patients with ascites: a study of 134 outpatients. Am J Gastroenterol 1993;88:514-519.

[9] Pugh RN, Murray-Lyon IM, Dawson JL, Pietroni MC, Williams R. Transection of the oesophagus for bleeding oesophageal varices. J Surg 1973;60:646-649. 
[10] Campion EW, deLabry LO, Glynn RJ. The effect of age on serum albumin in healthy males: report from the Normative Aging Study. J Gerontol 1988;43:M18-M20.

[11] Domenicali M, Baldassarre M, Giannone FA, Naldi M, Mastroroberto M, Biselli M, et al. Posttranscriptional changes of serum albumin: clinical and prognostic significance in hospitalized patients with cirrhosis. Hepatology 2014;60:1851-1860.

[12] Garcia-Martinez R, Caraceni P, Bernardi M, Ginés P, Arroyo V, Jalan R. Albumin: pathophysiologic basis of its role in the treatment of cirrhosis and its complications. Hepatology 2013;58:1836-1846.
[13] Curran-Everett D, Milgrom H. Post-hoc data analysis: benefits and limitations. Curr Opin Allergy Clin Immunol 2013;13:223224.

[14] Kent DM, Paulus JK, van Klaveren D, D'Agostino R, Goodman S, Hayward R, et al. The predictive approaches to treatment effect heterogeneity (PATH) statement. Ann Intern Med 2020;172:35-45.

[15] Steyerberg EW, Moons KG, van der Windt DA, Hayden JA, Perel P, Schroter S, et al. PROGRESS Group. Prognosis Research Strategy (PROGRESS) 3: prognostic model research. PLoS Med 2013;10: e1001381. 